Louisiana State University

LSU Digital Commons

$4-1-2015$

\title{
Strong Converse for the Classical Capacity of Optical Quantum Communication Channels
}

\author{
Bhaskar Roy Bardhan \\ Massachusetts Institute of Technology \\ Raul Garcia-Patron \\ Université Libre de Bruxelles \\ Mark M. Wilde \\ Louisiana State University \\ Andreas Winter \\ Universitat Autònoma de Barcelona
}

Follow this and additional works at: https://digitalcommons.Isu.edu/physics_astronomy_pubs

\section{Recommended Citation}

Bardhan, B., Garcia-Patron, R., Wilde, M., \& Winter, A. (2015). Strong Converse for the Classical Capacity of Optical Quantum Communication Channels. IEEE Transactions on Information Theory, 61 (4), 1842-1850. https://doi.org/10.1109/TIT.2015.2403840

This Article is brought to you for free and open access by the Department of Physics \& Astronomy at LSU Digital Commons. It has been accepted for inclusion in Faculty Publications by an authorized administrator of LSU Digital Commons. For more information, please contact ir@lsu.edu. 


\title{
Strong converse for the classical capacity of optical quantum communication channels
}

\author{
Bhaskar Roy Bardhan, Raúl García-Patrón, Mark M. Wilde, and Andreas Winter
}

\begin{abstract}
We establish the classical capacity of optical quantum channels as a sharp transition between two regimes-one which is an error-free regime for communication rates below the capacity, and the other in which the probability of correctly decoding a classical message converges exponentially fast to zero if the communication rate exceeds the classical capacity. This result is obtained by proving a strong converse theorem for the classical capacity of all phase-insensitive bosonic Gaussian channels, a well-established model of optical quantum communication channels, such as lossy optical fibers, amplifier and free-space communication. The theorem holds under a particular photonnumber occupation constraint, which we describe in detail in the paper. Our result bolsters the understanding of the classical capacity of these channels and opens the path to applications, such as proving the security of noisy quantum storage models of cryptography with optical links.
\end{abstract}

\section{Index Terms}

channel capacity, Gaussian quantum channels, optical communication channels, photon number constraint, strong converse theorem

\section{INTRODUCTION}

One of the most fundamental tasks in quantum information theory is to determine the ultimate limits on achievable data transmission rates for a noisy communication channel. The classical capacity is defined as the maximum rate at which it is possible to send classical data over a quantum channel such that the error probability decreases to zero in the limit of many independent uses of the channel [1], [2]. As such, the classical capacity serves as a distinctive bound on our ability to faithfully recover classical information sent over the channel.

The above definition of the classical capacity states that (a) for any rate below capacity, one can communicate with vanishing error probability in the limit of many channel uses and (b) there cannot exist such a communication scheme in the limit of many channel uses whenever the rate exceeds the capacity. However, strictly speaking, for any rate $R$ above capacity, the above definition leaves open the possibility for one to increase the communication rate $R$ by allowing for some error $\varepsilon>0$. Leaving room for the possibility of such a trade-off between the rate $R$ and the error $\varepsilon$ is the characteristic of a "weak converse,"

B. R. Bardhan is with the Hearne Institute for Theoretical Physics and Department of Physics and Astronomy, Louisiana State University, Baton Rouge, Louisiana 70803, USA (e-mail: broyba1@tigers.lsu.edu).

R. G-Patrón is with the Center for Quantum Information and Communication, Ecole Polytechnique de Bruxelles, CP 165, Universite Libre de Bruxelles, 1050, Bruxelles, Belgium (email: raulgarciapatron@gmail.com).

M. M. Wilde is with the Hearne Institute for Theoretical Physics, Department of Physics and Astronomy, Louisiana State University, Baton Rouge, Louisiana 70803, USA, and Center for Computation and Technology, Louisiana State University, Baton Rouge, Louisiana 70803, USA (email: mwilde@gmail.com).

A. Winter is with ICREA \& Física Teórica, Informació i Fenomens Quántics, Universitat Autònoma de Barcelona, ES-08193, Bellaterra (Barcelona), Spain (email: andreas.winter@uab.cat).

B. R. Bardhan and M. M. Wilde are grateful for support from the Department of Physics and Astronomy, Louisiana State University. M. M. Wilde was also supported by the DARPA Quiness Program through US Army Research Office award W31P4Q-12-1-0019. A. Winter acknowledges financial support by the Spanish MINECO, project FIS2008-01236 with the support of FEDER funds, the EC STREP "RAQUEL", the ERC Advanced Grant "IRQUAT", and the Philip Leverhulme Trust. R. G-Patrón acknowledges support from a Postdoctoral Researcher fellowship of the F.R.S.-FNRS and the action Back to Belgium Grants of the Belgian Federal Science Policy. R. G-Patrón also acknowledges financial support from the F.R.S.-FNRS under projects T.0199.13 and HIPERCOM, as well as from the Interuniversity Attraction Poles program of the Belgian Science Policy Office under Grant No. IAP P7-35 Photonics@ be.

This paper was presented in part at the IEEE International Symposium on Information Theory (ISIT) (2014).

Copyright (C) 2014 IEEE. Personal use of this material is permitted. 
and the corresponding capacity is sometimes called the weak capacity. A strong converse, on the contrary, establishes the capacity as a very sharp threshold, so that there is no such room for a trade-off between rate and error in the limit of many independent uses of the channel. That is, it guarantees that the error probability of any communication scheme asymptotically converges to one if its rate exceeds the classical capacity.

Despite their significance in understanding the ultimate information-carrying capacity of noisy communication channels, strong converse theorems are known to hold only for a handful of quantum channels: for those with classical inputs and quantum outputs [3], [4] (see earlier results for all classical channels [5], [6]), for all covariant channels with additive minimum output Rényi entropy [7], for all entanglementbreaking and Hadamard channels [8], as well as for pure-loss bosonic channels [9].

In this paper, we consider the encoding of classical messages into optical quantum states and the transmission of these codewords over phase-insensitive Gaussian channels. Phase-insensitive Gaussian channels are invariant with respect to phase-space rotations [10], [11], [12], [13], and they are considered to be one of the most practically relevant models to describe free space or optical fiber transmission, or transmission of classical messages through dielectric media, etc. In fact, phase-insensitive Gaussian channels constitute a broad class of noisy bosonic channels, encompassing all of the following: thermal noise channels (in which the signal photon states are mixed with a thermal state), additive noise channels (in which the input states are randomly displaced in phase space), and noisy amplifier channels [10], [14], [15], [16]. We prove that a strong converse theorem holds for the classical capacity of these channels, when imposing a photon-number occupation constraint on the inputs of the channel.

In some very recent studies [14], [17], [15], a solution to the long-standing minimum output entropy conjecture [12], [18] has been established for all phase-insensitive Gaussian channels, demonstrating that the minimum output entropy for such channels is indeed achieved by the vacuum input state. The major implication of this work is that we now know the classical capacity of any phase-insensitive Gaussian channel whenever there is a mean photon-number constraint on the channel inputs (the capacity otherwise being infinite if there is no photon number constraint). For instance, consider the thermal noise channel represented by a beamsplitter with transmissivity $\eta \in[0,1]$ mixing signaling photons (with mean photon number $N_{S}$ ) with a thermal state of mean photon number $N_{B}$. The results in [14], [17], [15] imply that the classical capacity of this channel is given by

$$
g\left(\eta N_{S}+(1-\eta) N_{B}\right)-g\left((1-\eta) N_{B}\right),
$$

where $g(x) \equiv(x+1) \log _{2}(x+1)-x \log _{2} x$ is the entropy of a bosonic thermal state with mean photon number $x$. However, the corresponding converse theorem, which can be inferred as a further implication of their work, is only a weak converse, in the sense that the upper bound on the communication rate $R$ of any coding scheme for the thermal noise channel can be written in the following form:

$$
R \leq \frac{1}{1-\varepsilon}\left[g\left(\eta N_{S}+(1-\eta) N_{B}\right)-g\left((1-\eta) N_{B}\right)+h_{2}(\varepsilon)\right]
$$

where $\varepsilon$ is the error probability, and $h_{2}(\varepsilon)$ is the binary entropy with the property that $\lim _{\varepsilon \rightarrow 0} h_{2}(\varepsilon)=0$. That is, only in the limit $\varepsilon \rightarrow 0$ does the above expression serve as the classical capacity of the channel, leaving room for a possible trade-off between rate and error probability. This observation also applies to the classical capacity of all other phase-insensitive Gaussian channels mentioned above.

In the present work, we prove that a strong converse theorem holds for the classical capacity of all phaseinsensitive Gaussian channels when imposing a photon-number occupation constraint. This means that if we demand that the average code density operator for the codewords, which are used for transmission of classical messages, is constrained to have a large shadow onto a subspace with photon number no larger than some fixed amount, then the probability of successfully decoding the message converges to zero in the limit of many channel uses if the rate $R$ of communication exceeds the classical capacity of these channels. We provide a mathematical definition in (32). 
This paper is structured as follows. In Section 【I, we review several preliminary ideas, including some definitions and notation for phase-insensitive Gaussian channels, and we recall structural decompositions of them that we exploit in our proof of the strong converse. We also recall the definition of the quantum Rényi entropy and an inequality that relates it to the smooth min-entropy [19]. In Section III, we then prove our main result, i.e., that the strong converse holds for the classical capacity of all phase-insensitive Gaussian channels when imposing a photon-number occupation constraint. We conclude with a brief summary in Section IV] along with suggestions for future research.

\section{PRELIMINARIES}

\section{A. Phase-insensitive Gaussian channels}

Bosonic Gaussian channels play a key role in modeling optical communication channels, such as optical fibers or free space transmission. They are represented by completely positive and trace preserving (CPTP) maps evolving Gaussian input states into Gaussian output states [20], [21], [11]. (A Gaussian state is completely characterized by a mean vector and a covariance matrix [20].) Single-mode Gaussian channels are characterized by two matrices $X$ and $Y$ acting on the covariance matrix $\Gamma$ of a single-mode Gaussian state in the following way:

$$
\Gamma \longrightarrow \Gamma^{\prime}=X \Gamma X^{T}+Y
$$

where $X^{T}$ is the transpose of the matrix $X$. Here $X$ and $Y$ are both $2 \times 2$ real matrices, satisfying

$$
Y \geq 0, \quad \operatorname{det} Y \geq(\operatorname{det} X-1)^{2},
$$

in order for the map to be a legitimate completely positive trace preserving map (see [20] for more details). A bosonic Gaussian quantum channel is said to be "quantum-limited' if the inequality above (involving $\operatorname{det} X$ and $\operatorname{det} Y$ ) is saturated [22], [13], [14], [15]. For instance, phase-insensitive Gaussian channels are quantum-limited when their environment is initially in a vacuum state.

In this work, we are interested in the most physically relevant set of phase-insensitive channels that corresponds to the choice

$$
\begin{aligned}
& X=\operatorname{diag}(\sqrt{\tau}, \sqrt{\tau}), \\
& Y=\operatorname{diag}(\nu, \nu),
\end{aligned}
$$

with $\tau, \nu \geq 0$ obeying the constraint above. The action of such phase-insensitive channels on an input signal mode can be uniquely described by their transformation of the symmetrically ordered characteristic function, defined as

$$
\chi(\mu) \equiv \operatorname{Tr}[\rho D(\mu)]
$$

where $D(\mu) \equiv \exp \left(\mu \hat{a}^{\dagger}-\mu^{*} \hat{a}\right)$ is the displacement operator for the input signal mode $\hat{a}$ [20]. For the Gaussian channels, the transformed characteristic function at the output is given by [15], [14], [21]

$$
\chi^{\prime}(\mu)=\chi(\sqrt{\tau} \mu) \exp \left(-\nu|\mu|^{2} / 2\right) .
$$

1) Examples: The canonical phase-insensitive Gaussian channels are the thermal noise channel, the additive noise channel, and the amplifier channel [16], [20], [14], [17], [10], [23], [18], [15].

The thermal channel $\mathcal{E}_{\eta, N_{B}}$ can be represented by a beamsplitter of transmissivity $\eta \in[0,1]$ that couples the input signal of mean photon number $N_{S}$ with a thermal state of mean photon number $N_{B}$. The special case $N_{B}=0$ corresponds to the pure-loss bosonic channel $\mathcal{E}_{\eta}$, where the state injected by the environment is the vacuum state.

In the additive noise channel $\mathcal{N}_{\bar{n}}$, each signal mode is randomly displaced in phase space according to a Gaussian distribution. The additive noise channel $\mathcal{N}_{\bar{n}}$ is completely characterized by the variance $\bar{n}$ of the noise introduced by the channel.

The quantum amplifier channel $\mathcal{A}_{G}^{N}$ is characterized by its gain $G \geq 1$ and the mean number of photons $N$ in the associated environment input mode (which is in a thermal state). The amplifier channel $\mathcal{A}_{G}^{N}$ is 
quantum-limited when the environment is in the vacuum state (we will denote such a quantum-limited amplifier by $\mathcal{A}_{G}^{0}$ ).

The transformed characteristic functions for these Gaussian channels are given by [17], [18], [16]

$$
\chi^{\prime}(\mu)= \begin{cases}\chi(\sqrt{\eta} \mu) e^{-(1-\eta)\left(N_{B}+1 / 2\right)|\mu|^{2}} & \text { for } \mathcal{E}_{\eta, N_{B}} \\ \chi(\mu) e^{-\bar{n}|\mu|^{2}} & \text { for } \mathcal{N}_{\bar{n}} \\ \chi(\sqrt{G} \mu) e^{-(G-1)(N+1 / 2)|\mu|^{2}} & \text { for } \mathcal{A}_{G}^{N} .\end{cases}
$$

2) Structural decompositions: Using the composition rule of Gaussian bosonic channels [24], any phase-insensitive Gaussian bosonic channel (let us denote it by $\mathcal{P}$ ) can be written as a concatenation of a pure-loss channel followed by a quantum-limited amplifier [10]

$$
\mathcal{P}=\mathcal{A}_{G}^{0} \circ \mathcal{E}_{T}
$$

where $\mathcal{E}_{T}$ is a pure-loss channel with parameter $T \in[0,1]$ and $\mathcal{A}_{G}^{0}$ is a quantum-limited amplifier with gain $G \geq 1$, these parameters chosen so that $\tau=T G$ and $\nu=G(1-T)+G-1$ (with $\tau$ and $\nu$ defined in (5)).

For instance, the additive noise channel $\mathcal{N}_{\bar{n}}$ can be realized as a pure-loss channel with transmissivity $T=1 /(\bar{n}+1)$ followed by a quantum-limited amplifier channel with gain $G=\bar{n}+1$. Also, we can consider the thermal noise channel $\mathcal{E}_{\eta, N_{B}}$ as a cascade of a pure-loss channel with transmissivity $T=\eta / G$ followed by a quantum-limited amplifier channel with gain $G=(1-\eta) N_{B}+1$. These two observations are equivalent to

$$
\begin{aligned}
\mathcal{N}_{\bar{n}}(\rho) & =\left(\mathcal{A}_{\bar{n}+1}^{0} \circ \mathcal{E}_{\overline{\bar{n}}+1}\right)(\rho), \\
\mathcal{E}_{\eta, N_{B}}(\rho) & =\left(\mathcal{A}_{(1-\eta) N_{B}+1}^{0} \circ \mathcal{E}_{T}\right)(\rho) .
\end{aligned}
$$

The above structural decompositions are useful in establishing the classical capacity as well as the minimum output entropy for all phase-insensitive channels [14], [17], [15].

3) Classical capacitites of phase-insensitive channels: Holevo, Schumacher, and Westmoreland (HSW) characterized the classical capacity of a quantum channel $\mathcal{N}$ in terms of a quantity now known as the Holevo information [1], [2]

$$
\chi(\mathcal{N}) \equiv \max _{\left\{p_{X}(x), \rho_{x}\right\}} I(X ; B)_{\rho},
$$

where $\left\{p_{X}(x), \rho_{x}\right\}$ represents an ensemble of quantum states, and the quantum mutual information $I(X ; B)_{\rho} \equiv H(X)_{\rho}+H(B)_{\rho}-H(X B)_{\rho}$, is defined with respect to a classical-quantum state $\rho_{X B} \equiv$ $\sum_{x} p_{X}(x)|x\rangle\left\langle\left. x\right|_{X} \otimes \mathcal{N}\left(\rho_{x}\right)_{B}\right.$. The above formula given by HSW for certain quantum channels is additive whenever

$$
\chi\left(\mathcal{N}^{\otimes n}\right)=n \chi(\mathcal{N}),
$$

for any positive integer $n$. For such quantum channels, the HSW formula is indeed equal to the classical capacity of those channels. However, a regularization is thought to be required in order to characterize the classical capacity of quantum channels for which the HSW formula cannot be shown to be additive. The classical capacity in general is then characterized by the following regularized formula:

$$
\chi_{\mathrm{reg}}(\mathcal{N}) \equiv \lim _{n \rightarrow \infty} \frac{1}{n} \chi\left(\mathcal{N}^{\otimes n}\right)
$$

The recent breakthrough works in [14], [15] (along with earlier results in [12], [25]) have established the following expressions for the classical capacities of various phase-insensitive channels:

$$
\begin{aligned}
C\left(\mathcal{E}_{\eta, N_{B}}\right) & =g\left(\eta N_{S}+(1-\eta) N_{B}\right)-g\left((1-\eta) N_{B}\right), \\
C\left(\mathcal{N}_{\bar{n}}\right) & =g\left(N_{S}+\bar{n}\right)-g(\bar{n}), \\
C\left(\mathcal{A}_{G}^{N}\right) & =g\left(G N_{S}+(G-1)(N+1)\right)-g((G-1)(N+1)),
\end{aligned}
$$


where $N_{S}$ is the mean input photon number. In general, the classical capacity of any phase-insensitive Gaussian channel is equal to

$$
g\left(N_{S}^{\prime}\right)-g\left(N_{B}^{\prime}\right)
$$

where $N_{S}^{\prime}=\tau N_{S}+(\tau+\nu-1) / 2$ and $N_{B}^{\prime}=(\tau+\nu-1) / 2$, with $\tau$ and $\nu$ defined in (5). In the above, $N_{S}^{\prime}$ is equal to the mean number of photons at the output when a thermal state of mean photon number $N_{S}$ is input, and $N_{B}^{\prime}$ is equal to the mean number of noise photons when the vacuum state is sent in. Note that the capacities in (15), (16), and (17) all have this particular form (but they differ in the corresponding mean number of photons). The classical capacities specified above are attainable by using coherent-state encoding schemes for the respective channels [12]. We will show in Section [II] that these expressions can also be interpreted as strong converse rates.

\section{B. Quantum Rényi entropy and smooth min-entropy}

The quantum Rényi entropy $H_{\alpha}(\rho)$ of a density operator $\rho$ is defined for $0<\alpha<\infty, \alpha \neq 1$ as

$$
H_{\alpha}(\rho) \equiv \frac{1}{1-\alpha} \log _{2} \operatorname{Tr}\left[\rho^{\alpha}\right] .
$$

It is a monotonic function of the " $\alpha$-purity" $\operatorname{Tr}\left[\rho^{\alpha}\right]$, and the von Neumann entropy $H(\rho)$ is recovered from it in the limit $\alpha \rightarrow 1$ :

$$
\lim _{\alpha \rightarrow 1} H_{\alpha}(\rho)=H(\rho) \equiv-\operatorname{Tr}\left[\rho \log _{2} \rho\right] .
$$

The min-entropy is recovered from it in the limit as $\alpha \rightarrow \infty$ :

$$
\lim _{\alpha \rightarrow \infty} H_{\alpha}(\rho)=H_{\min }(\rho) \equiv-\log _{2}\|\rho\|_{\infty}
$$

where $\|\rho\|_{\infty}$ is the infinity norm of $\rho$.

The quantum Rényi entropy of order $\alpha>1$ of a thermal state with mean photon number $N_{B}$ can be written as [26]

$$
\frac{\log _{2}\left[\left(N_{B}+1\right)^{\alpha}-N_{B}^{\alpha}\right]}{\alpha-1}
$$

For an additive noise channel $\mathcal{N}_{\bar{n}}$, the Rényi entropy $H_{\alpha}\left(\mathcal{N}_{\bar{n}}(\rho)\right)$ for $\alpha>1$ achieves its minimum value when the input $\rho$ is the vacuum state $|0\rangle\langle 0|[17]$ :

$$
\min _{\rho} H_{\alpha}\left(\mathcal{N}_{\bar{n}}(\rho)\right)=H_{\alpha}\left(\mathcal{N}_{\bar{n}}(|0\rangle\langle 0|)\right)=\frac{\log _{2}\left[(\bar{n}+1)^{\alpha}-\bar{n}^{\alpha}\right]}{\alpha-1} \text { for } \alpha>1 .
$$

Similarly, for the thermal noise channel $\mathcal{E}_{\eta, N_{B}}$, the Rényi entropy $H_{\alpha}\left(\mathcal{E}_{\eta, N_{B}}(\rho)\right)$ for $\alpha>1$ achieves its minimum value when the input $\rho$ is the vacuum state $|0\rangle\langle 0|$ [17]:

$$
\min _{\rho} H_{\alpha}\left(\mathcal{E}_{\eta, N_{B}}(\rho)\right)=H_{\alpha}\left(\mathcal{E}_{\eta, N_{B}}(|0\rangle\langle 0|)\right)=\frac{\log _{2}\left[\left((1-\eta) N_{B}+1\right)^{\alpha}-\left((1-\eta) N_{B}\right)^{\alpha}\right]}{\alpha-1} \text { for } \alpha>1 .
$$

In general, the main result of [17] shows that the minimum output Rényi entropy of any phase-insensitive Gaussian channel $\mathcal{P}$ is achieved by the vacuum state:

$$
\min _{\rho^{(n)}} H_{\alpha}\left(\mathcal{P}^{\otimes n}\left(\rho^{(n)}\right)\right)=n H_{\alpha}(\mathcal{P}(|0\rangle\langle 0|) .
$$

The above definition of the Rényi entropy can be generalized to the smooth Rényi entropy. This notion was first introduced by Renner and Wolf for classical probability distributions [19] and was later generalized to the quantum case (density operators). For a given density operator $\rho$, one can consider the set $\mathcal{B}^{\varepsilon}(\rho)$ of density operators $\tilde{\rho}$ that are $\varepsilon$-close to $\rho$ in trace distance for $\varepsilon \geq 0$ [27]. The $\varepsilon$-smooth quantum Rényi entropy of order $\alpha$ of a density operator $\rho$ is defined as [27]

$$
H_{\alpha}^{\varepsilon}(\rho) \equiv\left\{\begin{array}{cc}
\inf _{\tilde{\rho} \in \mathcal{B}^{\varepsilon}(\rho)} H_{\alpha}(\tilde{\rho}) & 0 \leq \alpha<1 \\
\sup _{\tilde{\rho} \in \mathcal{B}^{\varepsilon}(\rho)} H_{\alpha}(\tilde{\rho}) & 1<\alpha<\infty
\end{array} .\right.
$$


In the limit as $\alpha \rightarrow \infty$, we recover the smooth min-entropy of $\rho$ [27], [28]:

$$
H_{\min }^{\varepsilon}(\rho) \equiv \sup _{\tilde{\rho} \in \mathcal{B}^{\varepsilon}(\rho)}\left[-\log _{2}\|\widetilde{\rho}\|_{\infty}\right] .
$$

From the above, we see that the following relation holds

$$
\inf _{\widetilde{\rho} \in \mathcal{B}^{\varepsilon}(\rho)}\|\widetilde{\rho}\|_{\infty}=2^{-H_{\min }^{\varepsilon}(\rho)} .
$$

A relation between the smooth min-entropy and the Rényi entropy of order $\alpha>1$ is given by the following inequality [19]

$$
H_{\min }^{\varepsilon}(\rho) \geq H_{\alpha}(\rho)-\frac{1}{\alpha-1} \log _{2}\left(\frac{1}{\varepsilon}\right) .
$$

We will use this relation, along with the minimum output entropy results from [17], to prove the strong converse theorem for the classical capacity of all phase-insensitive Gaussian channels.

\section{STRONG CONVERSE FOR ALL PHASE-INSENSITIVE GAUSSIAN CHANNELS}

In this section, we consider the transmission of classical messages through phase-insensitive channels and show that a strong converse theorem holds for the classical capacity of these channels. Before doing so, we first make the following two observations:

- If the input signal states are allowed to have an arbitrarily large number of photons, then the classical capacity of the corresponding channel is infinite [12]. Thus, in order to have a sensible notion of the classical capacity for any quantum channel, one must impose some kind of constraint on the photon number of the states being fed into the channel. The most common kind of constraint is to demand that the mean number of photons in any signal transmitted through the channel can be at most $N_{S} \in[0, \infty)$. This is known as the mean photon number constraint and is commonly used in establishing the information-carrying capacity of a given channel [12], [25], [14], [15]. However, following the same arguments as in [9] (and later in [29]), we can show that the strong converse need not hold under such a mean photon number constraint. Indeed, as detailed in [9], there exists a communication scheme which allows for trading between communication rate and success probability, excluding the possibility of a strong converse holding under a mean photon number constraint. So instead, we prove that the strong converse theorem holds under a photon-number occupation constraint (see below for the definition and implication of this constraint) on the number of photons in the input states.

- Our proof of the strong converse theorem for the phase-insensitive channels can be regarded as a generalization of the arguments used in establishing the strong converse theorem for the classical capacity of the noiseless qubit channel [30], [7]. However, a comparison of our proof here and that for the noiseless qubit channel reveals that it is a significant generalization. Furthermore, our proof here also invites comparison with the proof of the strong converse for covariant channels with additive minimum output Rényi entropy [7], especially since additivity of minimum output Rényi entropies plays a critical role in the present paper.

Let $\rho_{m}$ denote a codeword of any code for communication over the phase-insensitive Gaussian channel $\mathcal{P}$. The photon-number occupation constraint that we impose on the codebook is to require that the average code density operator $\frac{1}{M} \sum_{m} \rho_{m}$ ( $M$ is the total number of messages) has a large shadow onto a subspace with photon number no larger than some fixed amount $n N_{S}$. Such a constraint on the channel inputs can be defined by introducing a photon number cutoff projector $\Pi_{L}$ that projects onto a subspace of $n$ bosonic modes such that the total photon number is no larger than $L$ :

$$
\Pi_{L} \equiv \sum_{a_{1}, \ldots, a_{n}: \sum_{i} a_{i} \leq L}\left|a_{1}\right\rangle\left\langle a_{1}|\otimes \ldots \otimes| a_{n}\right\rangle\left\langle a_{n}\right|,
$$


where $\left|a_{i}\right\rangle$ is a photon number state of photon number $a_{i}$. The rank of the above projector $\prod_{\left\lceil n N_{S}\right\rceil}$ has been shown to be never larger than $2^{n\left[g\left(N_{S}\right)+\delta_{0}\right]}$ (Lemma 3 in [9]), i.e.,

$$
\operatorname{Tr}\left\{\prod_{\left\lceil n N_{S}\right\rceil}\right\} \leq 2^{n\left[g\left(N_{S}\right)+\delta_{0}\right]},
$$

where $\delta_{0} \geq \frac{1}{n}\left(\log _{2} e+\log _{2}\left(1+\frac{1}{N_{S}}\right)\right)$, so that $\delta_{0}$ can be chosen arbitrarily small by taking $n$ large enough. Mathematically, the photon-number occupation constraint can then be written as

$$
\frac{1}{M} \sum_{m} \operatorname{Tr}\left\{\Pi_{\left\lceil n N_{S}\right\rceil} \rho_{m}\right\} \geq 1-\delta_{1}(n),
$$

where $\delta_{1}(n)$ is a function that decreases to zero as $n$ increases. In fact, the coherent-state encodings that attain the known capacities of the phase-insensitive channels do indeed satisfy the photon-number occupation constraint, with an exponentially decreasing $\delta_{1}(n)$, if coherent states with mean photon number per mode $<N_{S}-\delta$ are used, with $\delta$ being a small positive number (see Ref. [9] for an argument along these lines).

The first important step in proving the strong converse is to show that if most of the probability mass of the input state of the phase-insensitive channel $\mathcal{P}$ is in a subspace with photon number no larger than $n N_{S}$, then most of the probability mass of the channel output is in a subspace with photon number no larger than $n N_{S}^{\prime}$, where $N_{S}^{\prime}$ is the mean energy of the output state. We state this as the following lemma:

Lemma 1: Let $\rho^{(n)}$ denote a density operator on $n$ modes that satisfies

$$
\operatorname{Tr}\left\{\Pi_{\left\lceil n N_{S}\right\rceil} \rho^{(n)}\right\} \geq 1-\delta_{1}(n),
$$

where $\delta_{1}(n)$ is defined in (32). Let $\mathcal{P}$ be a phase-insensitive Gaussian channel with parameters $\tau$ and $\nu$ as defined in (5). Then

$$
\operatorname{Tr}\left\{\Pi_{\left.\left\lceil n N_{S}^{\prime}+\delta_{2}\right)\right\rceil} \mathcal{P}^{\otimes n}\left(\rho^{(n)}\right)\right\} \geq 1-\delta_{1}(n)-2 \sqrt{\delta_{1}(n)}-\delta_{3}(n),
$$

where $N_{S}^{\prime}=\tau N_{S}+(\tau+\nu-1) / 2, \mathcal{P}^{\otimes n}$ represents $n$ instances of $\mathcal{P}$ that act on the density operator $\rho^{(n)}$, $\delta_{2}$ is an arbitrarily small positive constant, and $\delta_{3}(n)$ is a function of $n$ decreasing to zero as $n \rightarrow \infty$.

Proof: The proof of this lemma is essentially the same as the proof of Lemma 1 of [29], with some minor modifications. We include the details of it for completeness. We first recall the structural decomposition in (9) for any phase-insensitive channel:

$$
\mathcal{P}(\rho)=\left(\mathcal{A}_{G}^{0} \circ \mathcal{E}_{T}\right)(\rho),
$$

i.e., that any phase-insensitive Gaussian channel can be realized as a concatenation of a pure-loss channel $\mathcal{E}_{T}$ of transmissivity $T$ followed by a quantum-limited amplifier channel $\mathcal{A}_{G}$ with gain $G$, with $\tau=T G$ and $\nu=G(1-T)+G-1$. Thus, a photon number state $|k\rangle\langle k|$ input to the phase-insensitive noise channel leads to an output of the following form:

$$
\mathcal{P}(|k\rangle\langle k|)=\sum_{m=0}^{k} p(m) \mathcal{A}_{G}^{0}(|m\rangle\langle m|),
$$

where

$$
p(m)=\left(\begin{array}{c}
k \\
m
\end{array}\right) T^{m}(1-T)^{k-m} .
$$

The quantum-limited amplifier channel has the following action on a photon number state $|m\rangle[10]$ :

$$
\mathcal{A}_{G}^{0}(|m\rangle\langle m|)=\sum_{l=0}^{\infty} q(l \mid m)|l\rangle\langle l|,
$$


where the conditional probabilities $q(l \mid m)$ are given by:

$$
q(l \mid m)=\left\{\begin{array}{cc}
0 & l<m \\
\left(1-\mu^{2}\right)^{m+1} \mu^{2(l-m)}\left(\begin{array}{c}
l \\
l-m
\end{array}\right) & l \geq m
\end{array},\right.
$$

where $\mu=\tanh r \in[0,1]$, with $r$ chosen such that $G=\cosh ^{2}(r)$.

The conditional distribution $q(l \mid m)$ has the two important properties of having finite second moment and exponential decay with increasing photon number. The property of exponential decay with increasing $l$ follows from

$$
\begin{aligned}
\left(1-\mu^{2}\right)^{m+1} \mu^{2(l-m)}\left(\begin{array}{c}
l \\
l-m
\end{array}\right) & =\left(1-\mu^{2}\right)^{m+1} \mu^{-2 m} 2^{-2 \log _{2}\left(\frac{1}{\mu}\right) l}\left(\begin{array}{c}
l \\
l-m
\end{array}\right) \\
& \leq\left(1-\mu^{2}\right)^{m+1} \mu^{-2 m} 2^{-2 \log _{2}\left(\frac{1}{\mu}\right) l} 2^{l h_{2}\left(\frac{l-m}{l}\right)} \\
& =\left(1-\mu^{2}\right)^{m+1} \mu^{-2 m} 2^{-l\left[2 \log _{2}\left(\frac{1}{\mu}\right)-h_{2}\left(\frac{l-m}{l}\right)\right]}
\end{aligned}
$$

The inequality applies the bound $\left(\begin{array}{l}n \\ k\end{array}\right) \leq 2^{n h_{2}(k / n)}$ (see (11.40) of [31]), where $h_{2}(x)$ is the binary entropy with the property that $\lim _{x \rightarrow 1} h_{2}(x)=0$. Thus, for large enough $l$, it will be the case that $2 \log \left(\frac{1}{\mu}\right)-$ $h_{2}\left(\frac{l-m}{l}\right)>0$, so that the conditional distribution $q(l \mid m)$ has exponential decay with increasing $l$. We can also then conclude that this distribution has a finite second moment. It follows from (36) that

$$
\mathcal{P}(|k\rangle\langle k|)=\sum_{l=0}^{\infty}\left[\sum_{m=0}^{k} p(m) q(l \mid m)\right]|l\rangle\langle l| .
$$

The eigenvalues above (i.e., $\sum_{m=0}^{k} p(m) q(l \mid m)$ ) represent a distribution over photon number states at the output of the phase-insensitive channel $\mathcal{P}$, which we can write as a conditional probability distribution $p(l \mid k)$ over $l$ given the input with definite photon number $k$. This probability distribution has its mean equal to $\tau k+(\tau+\nu-1) / 2$, since the mean energy of the input state is $k$. Furthermore, this distribution inherits the properties of having a finite second moment and an exponential decay to zero as $l \rightarrow \infty$.

For example, we can consider the thermal noise channel $\mathcal{E}_{\eta, N_{B}}$ with the structural decomposition given by (11)

$$
\mathcal{E}_{\eta, N_{B}}(\rho)=\left(\mathcal{A}_{(1-\eta) N_{B}+1}^{0} \circ \mathcal{E}_{\eta /\left((1-\eta) N_{B}+1\right)}\right)(\rho) .
$$

The mean of the corresponding distribution for this channel when a state of definite photon number $k$ is input, following the above arguments, is equal to $\eta k+(1-\eta) N_{B}$.

The argument from here is now exactly the same as the proof of Lemma 1 of [29] (starting from (40) of [29]). We include it here for completeness. We now suppose that the input state satisfies the photonnumber occupation constraint in (32), and apply the Gentle Measurement Lemma [32], [4] to obtain the following inequality

$$
\operatorname{Tr}\left\{\Pi_{\left\lceil n N_{S}^{\prime}+\delta_{2}\right\rceil} \mathcal{P}^{\otimes n}\left(\rho^{(n)}\right)\right\} \geq \operatorname{Tr}\left\{\Pi_{\left\lceil n N_{S}^{\prime}+\delta_{2}\right\rceil} \mathcal{P}^{\otimes n}\left(\Pi_{\left\lceil n N_{S}\right\rceil} \rho^{(n)} \Pi_{\left\lceil n N_{S}\right\rceil}\right)\right\}-2 \sqrt{\delta_{1}(n)},
$$

where $N_{S}^{\prime}=\tau N_{S}+(\tau+\nu-1) / 2$. Since there is photodetection at the output (i.e., the projector $\Pi_{\left\lceil n \eta N_{S}^{\prime}+\delta_{2}\right\rceil}$ is diagonal in the number basis), it suffices for us to consider the input $\Pi_{\left\lceil n N_{S}\right\rceil} \rho^{(n)} \prod_{\left\lceil n N_{S}\right\rceil}$ to be diagonal in the photon-number basis, and we write this as

$$
\rho^{(n)}=\sum_{a^{n}: \sum_{i} a_{i} \leq\left\lceil n N_{S}\right\rceil} p\left(a^{n}\right)\left|a^{n}\right\rangle\left\langle a^{n}\right|,
$$


where $\left|a^{n}\right\rangle$ represents strings of photon number states. We then find that (45) is equal to

$$
\begin{aligned}
\sum_{a^{n}: \sum_{i} a_{i} \leq\left\lceil n N_{S}\right\rceil} p\left(a^{n}\right) \operatorname{Tr}\left\{\left(\Pi_{\left\lceil n N_{S}^{\prime}+\delta_{2}\right\rceil}\right)\right. & \left.\mathcal{P}^{\otimes n}\left(\left|a^{n}\right\rangle\left\langle a^{n}\right|\right)\right\}-2 \sqrt{\delta_{1}(n)} \\
& =\sum_{a^{n}: \sum_{i} a_{i} \leq\left\lceil n N_{S}\right\rceil} p\left(a^{n}\right) \sum_{l^{n}: \sum_{i} l_{i} \leq\left\lceil n N_{S}^{\prime}+\delta_{2}\right\rceil} p\left(l^{n} \mid a^{n}\right)-2 \sqrt{\delta_{1}(n)},
\end{aligned}
$$

where the distribution $p\left(l^{n} \mid a^{n}\right) \equiv \prod_{i=1}^{n} p\left(l_{i} \mid a_{i}\right)$ with $p\left(l_{i} \mid a_{i}\right)$ coming from (43).

In order to obtain a lower bound on the expression in (47), we analyze the term

$$
\sum_{l^{n}: \sum_{i} l_{i} \leq\left\lceil n N_{S}^{\prime}+\delta_{2}\right\rceil} p\left(l^{n} \mid a^{n}\right)
$$

on its own under the assumption that $\sum_{i} a_{i} \leq\left\lceil n N_{S}\right\rceil$. Let $L_{i} \mid a_{i}$ denote a conditional random variable with distribution $p\left(l_{i} \mid a_{i}\right)$, and let $\overline{L^{n}} \mid a^{n}$ denote the sum random variable:

$$
\overline{L^{n}}\left|a^{n} \equiv \sum_{i} L_{i}\right| a_{i}
$$

so that

$$
\begin{aligned}
\sum_{l^{n}: \sum_{i} l_{i} \leq\left\lceil n N_{S}^{\prime}+\delta_{2}\right\rceil} p\left(l^{n} \mid a^{n}\right) & =\operatorname{Pr}\left\{\overline{L^{n}} \mid a^{n} \leq n\left(N_{S}^{\prime}+\delta_{2}\right)\right\} \\
& =\operatorname{Pr}\left\{\overline{L^{n}} \mid a^{n} \leq n\left(\tau N_{S}+(\tau+\nu-1) / 2+\delta_{2}\right)\right\} \\
& \geq \operatorname{Pr}\left\{\overline{L^{n}} \mid a^{n} \leq n\left(\tau \frac{1}{n} \sum_{i} a_{i}+(\tau+\nu-1) / 2+\delta_{2}\right)\right\},
\end{aligned}
$$

where $(\tau+\nu-1) / 2$ represents the mean number of noise photons injected by the channel, and the inequality follows from the constraint $\sum_{i} a_{i} \leq\left\lceil n N_{S}\right\rceil$. Since

$$
\mathbb{E}\left\{L_{i} \mid a_{i}\right\}=\tau a_{i}+(\tau+\nu-1) / 2,
$$

it follows that

$$
\mathbb{E}\left\{\overline{L^{n}} \mid a^{n}\right\}=n\left(\tau \frac{1}{n} \sum_{i} a_{i}+(\tau+\nu-1) / 2\right),
$$

and so the expression in (52) is the probability that a sum of independent random variables deviates from its mean by no more than $\delta_{2}$. To obtain a bound on the probability in (52) from below, we now follow the approach in [29] employing the truncation method (see Section 2.1 of [33] for more details), in which each random variable $L_{i} \mid a_{i}$ is split into two parts:

$$
\begin{aligned}
& \left(L_{i} \mid a_{i}\right)_{>T_{0}} \equiv\left(L_{i} \mid a_{i}\right) \mathcal{I}\left(\left(L_{i} \mid a_{i}\right)>T_{0}\right), \\
& \left(L_{i} \mid a_{i}\right)_{\leq T_{0}} \equiv\left(L_{i} \mid a_{i}\right) \mathcal{I}\left(\left(L_{i} \mid a_{i}\right) \leq T_{0}\right),
\end{aligned}
$$

where $\mathcal{I}(\cdot)$ is the indicator function and $T_{0}$ is a truncation parameter taken to be very large (much larger than $\max _{i} a_{i}$, for example). We can then split the sum random variable into two parts as well:

$$
\begin{aligned}
\overline{L^{n}} \mid a^{n} & =\left(\overline{L^{n}} \mid a^{n}\right)_{>T_{0}}+\left(\overline{L^{n}} \mid a^{n}\right)_{\leq T_{0}} \\
& \equiv \sum_{i}\left(L_{i} \mid a_{i}\right)_{>T_{0}}+\sum_{i}\left(L_{i} \mid a_{i}\right)_{\leq T_{0}} .
\end{aligned}
$$


We can use the union bound to argue that

$$
\begin{aligned}
\operatorname{Pr}\left\{\overline{L^{n}} \mid a^{n} \geq \mathbb{E}\left\{\overline{L^{n}} \mid a^{n}\right\}+n \delta_{2}\right\} \leq \operatorname{Pr}\left\{\left(\overline{L^{n}} \mid a^{n}\right)_{>T_{0}} \geq \mathbb{E}\left\{\left(\overline{L^{n}} \mid a^{n}\right)_{>T_{0}}\right\}+n \delta_{2} / 2\right\} \\
+\operatorname{Pr}\left\{\left(\overline{L^{n}} \mid a^{n}\right)_{\leq T_{0}} \geq \mathbb{E}\left\{\left(\overline{L^{n}} \mid a^{n}\right)_{\leq T_{0}}\right\}+n \delta_{2} / 2\right\} .
\end{aligned}
$$

The idea from here is that for a random variable $L_{i} \mid a_{i}$ with sufficient decay for large values, we can bound the first probability for $\left(\overline{L^{n}} \mid a^{n}\right)_{>T_{0}}$ from above by $\varepsilon / \delta_{2}$ for $\varepsilon$ an arbitrarily small positive constant (made small by taking $T_{0}$ larger) by employing the Markov inequality. We then bound the second probability for $\left(\overline{L^{n}} \mid a^{n}\right)_{<T}$ using a Chernoff bound, since these random variables are bounded. This latter bound has an exponential decay with increasing $n$ due to the ability to use a Chernoff bound. So, the argument is just to make $\varepsilon$ arbitrarily small by increasing the truncation parameter $T_{0}$, and for $n$ large enough, exponential convergence to zero kicks in. We point the reader to Section 2.1 of [33] for more details. By using either approach, we arrive at the following bound:

$$
\sum_{l^{n}: \sum_{i} l_{i} \leq\left\lceil n N_{S}^{\prime}+\delta_{2}\right\rceil} p\left(l^{n} \mid a^{n}\right) \geq 1-\delta_{3}(n),
$$

where $\delta_{3}(n)$ is a function decreasing to zero as $n \rightarrow \infty$. Finally, we put this together with (47) to obtain

$$
\begin{aligned}
& \operatorname{Tr}\left\{\prod_{\left\lceil n N_{S}^{\prime}+\delta_{2}\right\rceil^{-}} \mathcal{P}^{\otimes n}\left(\rho^{(n)}\right)\right\} \\
& \geq \sum_{a^{n}: \sum_{i} a_{i} \leq\left\lceil n N_{S}\right\rceil} p\left(a^{n}\right) \sum_{l^{n}: \sum_{i} l_{i} \leq\left\lceil n N_{S}^{\prime}+\delta_{2}\right\rceil} p\left(l^{n} \mid a^{n}\right)-2 \sqrt{\delta_{1}(n)} \\
& \geq\left(1-\delta_{1}(n)\right)\left(1-\delta_{3}(n)\right)-2 \sqrt{\delta_{1}(n)} \\
& \geq 1-\delta_{1}(n)-\delta_{3}(n)-2 \sqrt{\delta_{1}(n)},
\end{aligned}
$$

thereby completing the proof.

Let $\Lambda_{m}$ denote a decoding POVM acting on the output space of $n$ instances of the phase-insensitive channel. In what follows, we prove the strong converse theorem for the classical capacity of all phaseinsensitive Gaussian channels.

Theorem 1: Let $\mathcal{P}$ be a phase-insensitive Gaussian channel with parameters $\tau$ and $\nu$ as defined in (5). The average success probability $p_{\text {succ }}$ of any code for this channel satisfying (32) is bounded as

$$
p_{\text {succ }}=\frac{1}{M} \sum_{m} \operatorname{Tr}\left\{\Lambda_{m} \mathcal{P}^{\otimes n}\left(\rho_{m}\right)\right\} \leq 2^{-n R_{2}} 2^{\left[g\left(N_{S}^{\prime}\right)-H_{\alpha}(\mathcal{P}(|0\rangle\langle 0|))+\delta_{2}+\frac{1}{n(\alpha-1)} \log _{2}(1 / \varepsilon)\right]}+\varepsilon+\delta_{6}(n),
$$

where $\alpha>1, \varepsilon \in(0,1), N_{S}^{\prime}=\tau N_{S}+(\tau+\nu-1) / 2, \mathcal{P}^{\otimes n}$ denotes $n$ instances of $\mathcal{P}, \delta_{1}(n)$ is defined in (32), $\delta_{2}$ is an arbitrarily small positive constant, $\delta_{3}(n)$ is a function decreasing with $n$ (both defined in Lemma 1), and $\delta_{6}(n)=2 \sqrt{\delta_{1}(n)+2 \sqrt{\delta_{1}(n)}+\delta_{3}(n)}$.

Proof: This proof is very similar to the proof of Theorem 2 of [29], with the exception that we can now invoke the main result of [17] (that the minimum output entropy for Rényi entropies of arbitrary order is attained by the vacuum state input). Consider the success probability of any code satisfying the 
photon-number occupation constraint (32):

$$
\begin{aligned}
\frac{1}{M} \sum_{m} \operatorname{Tr}\left\{\Lambda_{m} \mathcal{P}^{\otimes n}\left(\rho_{m}\right)\right\} \leq & \frac{1}{M} \sum_{m} \operatorname{Tr}\left\{\Lambda_{m} \Pi_{\left\lceil n N_{S}^{\prime}\right]} \mathcal{P}^{\otimes n}\left(\rho_{m}\right) \Pi_{\left[n N_{S}^{\prime}\right]}\right\} \\
& +\frac{1}{M} \sum_{m}\left\|\Pi_{\left\lceil n N_{S}^{\prime}\right]} \mathcal{P}^{\otimes n}\left(\rho_{m}\right) \Pi_{\left\lceil n N_{S}^{\prime}\right\rceil}-\mathcal{P}^{\otimes n}\left(\rho_{m}\right)\right\|_{1} \\
\leq & \frac{1}{M} \sum_{m} \operatorname{Tr}\left\{\Lambda_{m} \Pi_{\left\lceil n N_{S}^{\prime}\right]} \mathcal{P}^{\otimes n}\left(\rho_{m}\right) \Pi_{\left[n N_{S}^{\prime}\right]}\right\} \\
& +2 \sqrt{\delta_{1}(n)+2 \sqrt{\delta_{1}(n)}+\delta_{3}(n)} .
\end{aligned}
$$

The first inequality is a special case of the inequality

$$
\operatorname{Tr}\{\Lambda \sigma\} \leq \operatorname{Tr}\{\Lambda \rho\}+\|\rho-\sigma\|_{1}
$$

which holds for $0 \leq \Lambda \leq I, \rho, \sigma \geq 0$, and $\operatorname{Tr}\{\rho\}, \operatorname{Tr}\{\sigma\} \leq 1$. The second inequality is obtained by invoking Lemma 1 and the Gentle Measurement Lemma [32], [4] for ensembles.

Note that in the above, the second term vanishes as $n \rightarrow \infty$; hence it suffices to focus on the first term, which by cyclicity of trace yields

$$
\frac{1}{M} \sum_{m} \operatorname{Tr}\left\{\Lambda_{m} \Pi_{\left[n N_{S}^{\prime}\right]} \mathcal{P}^{\otimes n}\left(\rho_{m}\right) \Pi_{\left\lceil n N_{S}^{\prime}\right]}\right\}=\frac{1}{M} \sum_{m} \operatorname{Tr}\left\{\Pi_{\left[n N_{S}^{\prime}\right\rceil} \Lambda_{m} \Pi_{\left\lceil n N_{S}^{\prime}\right]} \mathcal{P}^{\otimes n}\left(\rho_{m}\right)\right\} .
$$

At this point, we consider the set of all states $\widetilde{\sigma}_{m}$ that are $\varepsilon$-close in trace distance to each output of the phase-insensitive channel $\mathcal{P}^{\otimes n}\left(\rho_{m}\right)$ (let us denote this set by $\mathcal{B}^{\varepsilon}\left(\mathcal{P}^{\otimes n}\left(\rho_{m}\right)\right)$. This consideration will allow us to relate the success probability to the smooth min-entropy. We find the following upper bound on (69):

$$
\begin{aligned}
\frac{1}{M} \sum_{m} \operatorname{Tr}\left\{\Pi_{\left\lceil n N_{S}^{\prime}\right\rceil} \Lambda_{m} \Pi_{\left\lceil n N_{S}^{\prime}\right\rceil} \mathcal{P}^{\otimes n}\left(\rho_{m}\right)\right\} & \leq \frac{1}{M} \sum_{m} \operatorname{Tr}\left\{\Pi_{\left\lceil n N_{S}^{\prime}\right\rceil} \Lambda_{m} \Pi_{\left\lceil n N_{S}^{\prime}\right\rceil} \widetilde{\sigma}_{m}\right\}+\varepsilon \\
& \leq \frac{1}{M} \sum_{m} \operatorname{Tr}\left\{\Pi_{\left\lceil n N_{S}^{\prime}\right\rceil} \Lambda_{m} \Pi_{\left\lceil n N_{S}^{\prime}\right\rceil}\right\}\left\|\widetilde{\sigma}_{m}\right\|_{\infty}+\varepsilon .
\end{aligned}
$$

We can now optimize over all of the states $\widetilde{\sigma}_{m}$ that are $\varepsilon$-close to $\mathcal{P}^{\otimes n}\left(\rho_{m}\right)$, leading to the tightest upper bound on the success probability

$$
\begin{aligned}
\frac{1}{M} \sum_{m} \operatorname{Tr}\left\{\Pi_{\left\lceil n N_{S}^{\prime}\right\rceil} \Lambda_{m} \Pi_{\left\lceil n N_{S}^{\prime}\right]} \mathcal{P}^{\otimes n}\left(\rho_{m}\right)\right\} & \\
\leq & \frac{1}{M} \sum_{m} \operatorname{Tr}\left\{\Pi_{\left\lceil n N_{S}^{\prime}\right\rceil} \Lambda_{m} \Pi_{\left\lceil n N_{S}^{\prime}\right\rceil}\right\} \inf _{\widetilde{\sigma}_{m} \in \mathcal{B}^{\varepsilon}\left(\mathcal{P} \otimes n\left(\rho_{m}\right)\right)}\left\|\widetilde{\sigma}_{m}\right\|_{\infty}+\varepsilon .
\end{aligned}
$$

Since the quantity $\inf _{\widetilde{\sigma}_{m} \in \mathcal{B}^{\varepsilon}\left(\mathcal{P} \otimes n\left(\rho_{m}\right)\right)}\left\|\widetilde{\sigma}_{m}\right\|_{\infty}$ is related to the smooth min-entropy via

$$
\inf _{\widetilde{\sigma}_{m} \in \mathcal{B}^{\varepsilon}\left(\mathcal{P}^{\otimes n}\left(\rho_{m}\right)\right)}\left\|\widetilde{\sigma}_{m}\right\|_{\infty}=2^{-H_{\min }^{\varepsilon}\left(\mathcal{P}^{\otimes n}\left(\rho_{m}\right)\right)},
$$

the upper bound in (72) gives

$$
\begin{aligned}
& \frac{1}{M} \sum_{m} \operatorname{Tr}\left\{\Pi_{\left\lceil n N_{S}^{\prime}\right\rceil} \Lambda_{m} \Pi_{\left\lceil n N_{S}^{\prime}\right\rceil}\right\} 2^{-H_{\min }^{\varepsilon}\left(\mathcal{P}^{\otimes n}\left(\rho_{m}\right)\right)}+\varepsilon \\
& \leq \frac{1}{M} \sum_{m} \operatorname{Tr}\left\{\Pi_{\left\lceil n N_{S}^{\prime}\right\rceil} \Lambda_{m} \Pi_{\left\lceil n N_{S}^{\prime}\right\rceil}\right\} \sup _{\rho} 2^{-H_{\min }^{\varepsilon}\left(\mathcal{P}^{\otimes n}(\rho)\right)}+\varepsilon \\
& =\frac{1}{M} 2^{-\inf _{\rho} H_{\min }^{\varepsilon}\left(\mathcal{P}^{\otimes n}(\rho)\right)} \operatorname{Tr}\left\{\Pi_{\left\lceil n N_{S}^{\prime}\right]}\right\}+\varepsilon \\
& \leq 2^{-n R} 2^{-\inf _{\rho} H_{\min }^{\varepsilon}\left(\mathcal{P}^{\otimes n}(\rho)\right)} 2^{n\left[g\left(N_{S}^{\prime}\right)+\delta\right]}+\varepsilon .
\end{aligned}
$$


The first inequality follows by taking a supremum over all input states. The first equality follows because $\sum_{m} \Lambda_{m}=I$ for the set of decoding POVM measurements $\left\{\Lambda_{m}\right\}$, and the second inequality is a result of the upper bound on the rank of the photon number cutoff projector in (31). We have also used the fact that the rate of the channel is expressed as $R=\left(\log _{2} M\right) / n$, where $M$ is the number of messages.

Observe that the success probability is now related to the smooth min-entropy, and we can exploit the following relation between smooth min-entropy and the Rényi entropies for $\alpha>1$ [19]:

$$
H_{\min }^{\varepsilon}(\omega) \geq H_{\alpha}(\omega)-\frac{1}{\alpha-1} \log _{2}\left(\frac{1}{\varepsilon}\right) .
$$

Using the above inequality and the fact that the "strong" Gaussian optimizer conjecture has been proven for the Rényi entropies of all orders [17] (recall (25)), we get that

$$
\inf _{\rho} H_{\min }^{\varepsilon}\left(\mathcal{P}^{\otimes n}(\rho)\right) \geq n\left[H_{\alpha}(\mathcal{P}(|0\rangle\langle 0|))-\frac{1}{n(\alpha-1)} \log _{2}\left(\frac{1}{\varepsilon}\right)\right] .
$$

The first term on the right hand side is a result of the fact that the vacuum state minimizes the Rényi entropy of all orders at the output of a phase-insensitive Gaussian channel.

By tuning the parameters $\alpha$ and $\varepsilon$ appropriately, we recover the strong converse theorem:

Corollary 1 (Strong converse): Let $\mathcal{P}$ be a phase-insensitive Gaussian channel with parameters $\tau$ and $\nu$ as defined in (5). The average success probability $p_{\text {succ }}$ of any code for this channel satisfying (32) is bounded as

$$
p_{\text {succ }}=\frac{1}{M} \sum_{m} \operatorname{Tr}\left\{\Lambda_{m} \mathcal{P}^{\otimes n}\left(\rho_{m}\right)\right\} \leq 2^{-n R} 2^{n\left[g\left(N_{S}^{\prime}\right)-g\left(N_{B}^{\prime}\right)+\delta_{2}+\delta_{5} / \delta_{4}+\delta_{4} K\left(N_{B}^{\prime}\right)\right]}+2^{-n \delta_{5}}+\delta_{6}(n),
$$

where $N_{S}^{\prime}=\tau N_{S}+(\tau+\nu-1) / 2, N_{B}^{\prime} \equiv(\tau+\nu-1) / 2, \mathcal{P}^{\otimes n}$ denotes $n$ instances of $\mathcal{P}, \delta_{1}(n)$ is defined in (32), $\delta_{2}$ is an arbitrarily small positive constant, $\delta_{3}(n)$ is a function decreasing with $n$ (both defined in Lemma 1), $\delta_{4}$ and $\delta_{5}$ are arbitrarily small positive constants such that $\delta_{5} / \delta_{4}$ is arbitrarily small, and $K\left(N_{B}^{\prime}\right)$ is a function of $N_{B}^{\prime}$ only. Also, $\delta_{6}(n)=2 \sqrt{\delta_{1}(n)+2 \sqrt{\delta_{1}(n)}+\delta_{3}(n)}$. Thus, for any rate $R>g\left(N_{S}^{\prime}\right)-g\left(N_{B}^{\prime}\right)$, it is possible to choose the parameters such that the success probability of any family of codes satisfying (32) decreases to zero in the limit of large $n$.

Proof: In Theorem 1, we can pick $\alpha=1+\delta_{4}$ and $\varepsilon=2^{-n \delta_{5}}$, with $\delta_{5}>0$ much smaller than $\delta_{4}>0$ such that $\delta_{5} / \delta_{4}$ is arbitrarily small, and the terms on the right hand side in (76) simplify to

$$
n\left[H_{1+\delta_{4}}(\mathcal{P}(|0\rangle\langle 0|))-\frac{\delta_{5}}{\delta_{4}}\right] .
$$

The output state $\mathcal{P}(|0\rangle\langle 0|)$ for the phase-insensitive channel with the vacuum state as the input is a thermal state with mean photon number $N_{B}^{\prime} \equiv(\tau+\nu-1) / 2$. The quantum Rényi entropy of order $\alpha>1$ of a thermal state with mean photon number $N_{B}^{\prime}$ is given by [18]

$$
\frac{\log _{2}\left[\left(N_{B}^{\prime}+1\right)^{\alpha}-N_{B}^{\prime \alpha}\right]}{\alpha-1} \text {. }
$$

Lemma 6.3 of [28] gives us the following inequality for a general state (for $\alpha$ close enough to one):

$$
H_{\alpha}(\rho) \geq H(\rho)-4(\alpha-1)\left(\log _{2} v\right)^{2},
$$

where

$$
v \equiv 2^{-\frac{1}{2} H_{3 / 2}(\rho)}+2^{\frac{1}{2} H_{1 / 2}(\rho)}+1 .
$$


For a thermal state, we find using (79) that

$$
\begin{aligned}
& H_{3 / 2}(\rho)=2 \log _{2}\left[\left(N_{B}^{\prime}+1\right)^{3 / 2}-N_{B}^{\prime 3 / 2}\right], \\
& H_{1 / 2}(\rho)=-2 \log _{2}\left[\left(N_{B}^{\prime}+1\right)^{1 / 2}-N_{B}^{\prime 1 / 2}\right],
\end{aligned}
$$

so that

$$
v\left(N_{B}^{\prime}\right)=\left[\left(N_{B}^{\prime}+1\right)^{3 / 2}-N_{B}^{\prime 3 / 2}\right]^{2}+\left[\left(N_{B}^{\prime}+1\right)^{1 / 2}-N_{B}^{\prime 1 / 2}\right]^{-2}+1 .
$$

We then find that

$$
\begin{aligned}
H_{1+\delta_{4}}(\mathcal{P}(|0\rangle\langle 0|)) & \geq H(\mathcal{P}(|0\rangle\langle 0|))-\delta_{4} K\left(N_{B}^{\prime}\right) \\
& =g\left(N_{B}^{\prime}\right)-\delta_{4} K\left(N_{B}^{\prime}\right),
\end{aligned}
$$

where

$$
K\left(N_{B}^{\prime}\right) \equiv 4\left[\log _{2} v\left(N_{B}^{\prime}\right)\right]^{2} .
$$

We now recover the bound in the statement of the corollary.

Finally, we recall the capacities of the phase-insensitive channels in (15), (16), and (17). Comparing them with the statement of Corollary 1, we can conclude that these expressions indeed represent strong converse rates for these respective channels, since the success probability when communicating above these rates decreases to zero in the limit $n \rightarrow \infty$.

\section{CONCLUSION}

Phase-insensitive Gaussian channels represent physical noise models which are relevant for optical communication, including attenuation, thermalization, or amplification of optical signals. In this paper, we combine the proofs in [29] with the recent results of [14], [17], [15] to prove that a strong converse theorem holds for the classical capacity of all phase-insensitive Gaussian quantum channels. We showed that the success probability of correctly decoding classical information asymptotically converges to zero in the limit of many channel uses, if the communication rate exceeds the capacity. Our result thus establishes the capacity of these channels as a very sharp dividing line between possible and impossible communication rates through these channels. This result might find an immediate application in proving security of noisy quantum storage models of cryptography [34] for continuous-variable systems. The results of this paper can also be easily extended to the more general case of multimode bosonic Gaussian channels [14].

As an open question, one might attempt to establish a strong converse for the classical capacity of all phase-sensitive Gaussian channels. Another area of research where our result might be extended is in the setting of network information theory-for example, one might consider establishing a strong converse for the classical capacity of the multiple-access bosonic channels, in which two or more senders communicate to a common receiver over a shared communication channel [35].

\section{REFERENCES}

[1] A. S. Holevo, "The capacity of the quantum channel with general signal states," IEEE Transactions on Information Theory, vol. 44, pp. 269-273, January 1998, arXiv:quant-ph/9611023.

[2] B. Schumacher and M. D. Westmoreland, "Sending classical information via noisy quantum channels," Physical Review A, vol. 56, pp. 131-138, July 1997. [Online]. Available: http://link.aps.org/doi/10.1103/PhysRevA.56.131

[3] T. Ogawa and H. Nagaoka, "Strong converse to the quantum channel coding theorem," IEEE Transactions on Information Theory, vol. 45, pp. 2486-2489, November 1999, arXiv:quant-ph/9808063.

[4] A. Winter, "Coding theorem and strong converse for quantum channels," IEEE Transactions on Information Theory, vol. 45, no. 7, pp. 2481-2485, 1999.

[5] J. Wolfowitz, Coding Theorems of Information Theory. Springer, 1964, vol. 31.

[6] S. Arimoto, "On the converse to the coding theorem for discrete memoryless channels," IEEE Transactions on Information Theory, vol. 19, pp. 357-359, May 1973.

[7] R. Koenig and S. Wehner, "A strong converse for classical channel coding using entangled inputs," Physical Review Letters, vol. 103, p. 070504, August 2009, arXiv:0903.2838. 
[8] M. M. Wilde, A. Winter, and D. Yang, "Strong converse for the classical capacity of entanglement-breaking and Hadamard channels via a sandwiched rényi relative entropy," Communications in Mathematical Physics, vol. 331, no. 2, pp. 593-622, October 2014, arXiv:1306.1586.

[9] M. M. Wilde and A. Winter, "Strong converse for the classical capacity of the pure-loss bosonic channel," Problems of Information Transmission, vol. 50, no. 2, pp. 117-132, Jul. 2014, arXiv:1308.6732. [Online]. Available: http://link.springer.com/10.1134/S003294601402001X

[10] R. Garcia-Patron, C. Navarrete-Benlloch, S. Lloyd, J. H. Shapiro, and N. J. Cerf, "Majorization theory approach to the Gaussian channel minimum entropy conjecture," Physical Review Letters, vol. 108, p. 110505, March 2012, arXiv:1111.1986. [Online]. Available: http://link.aps.org/doi/10.1103/PhysRevLett.108.110505

[11] C. M. Caves and P. D. Drummond, "Quantum limits on bosonic communication rates," Reviews of Modern Physics, vol. 66, pp. 481-537, April 1994. [Online]. Available: http://link.aps.org/doi/10.1103/RevModPhys.66.481

[12] A. S. Holevo and R. F. Werner, "Evaluating capacities of bosonic Gaussian channels," Physical Review A, vol. 63, p. 032312, February 2001, arXiv:quant-ph/9912067. [Online]. Available: http://link.aps.org/doi/10.1103/PhysRevA.63.032312

[13] J. S. Ivan, K. K. Sabapathy, and R. Simon, "Operator-sum representation for bosonic Gaussian channels," Physical Review A, vol. 84, p. 042311, October 2011, arXiv:1012.4266. [Online]. Available: http://link.aps.org/doi/10.1103/PhysRevA.84.042311

[14] V. Giovannetti, A. S. Holevo, and R. García-Patrón, "A solution of the Gaussian optimizer conjecture for quantum channels," Accepted into Communications in Mathematical Physics, August 2014, arXiv:1312.2251.

[15] V. Giovannetti, R. Garcia-Patron, N. J. Cerf, and A. S. Holevo, "Ultimate classical communication rates of quantum optical channels," Nature Photonics, vol. 8, pp. 796-800, September 2014, arXiv:1312.6225.

[16] V. Giovannetti, S. Lloyd, L. Maccone, and J. H. Shapiro, "Electromagnetic channel capacity for practical purposes," Nature Photonics, vol. 7, no. 10, pp. 834-838, October 2013, arXiv:1210.3300. [Online]. Available: http://dx.doi.org/10.1038/nphoton.2013.193

[17] A. Mari, V. Giovannetti, and A. S. Holevo, "Quantum state majorization at the output of bosonic Gaussian channels," Nature Communications, vol. 5, no. 3826, May 2014, arXiv:1312.3545.

[18] V. Giovannetti, S. Guha, S. Lloyd, L. Maccone, and J. H. Shapiro, "Minimum output entropy of bosonic channels: A conjecture," Physical Review A, vol. 70, p. 032315, September 2004, arXiv:quant-ph/0404005. [Online]. Available: http://link.aps.org/doi/10.1103/PhysRevA.70.032315

[19] R. Renner and S. Wolf, "Smooth Rényi entropy and applications," in Proceedings of the 2007 International Symposium on Information Theory, 2004, p. 232. [Online]. Available: http://www.ti.inf.ethz.ch/sw/publications/smooth.ps

[20] C. Weedbrook, S. Pirandola, R. García-Patrón, N. J. Cerf, T. C. Ralph, J. H. Shapiro, and S. Lloyd, "Gaussian quantum information," Reviews of Modern Physics, vol. 84, pp. 621-669, May 2012, arXiv:1110.3234.

[21] J. Eisert and M. M. Wolf, "Gaussian quantum channels," Quantum Information with Continuous Variables of Atoms and Light, pp. 23-42, 2007, arXiv:quant-ph/0505151.

[22] C. M. Caves, "Quantum limits on noise in linear amplifiers," Physical Review D, vol. 26, pp. 1817-1839, October 1982. [Online]. Available: http://link.aps.org/doi/10.1103/PhysRevD.26.1817

[23] J. Schafer, E. Karpov, R. Garcia-Patron, O. V. Pilyavets, and N. J. Cerf, "Equivalence relations for the classical capacity of single-mode Gaussian quantum channels," Physical Review Letters, vol. 111, p. 030503, July 2013, arXiv:1303.4939. [Online]. Available: http://link.aps.org/doi/10.1103/PhysRevLett.111.030503

[24] F. Caruso, V. Giovannetti, and A. S. Holevo, "One-mode bosonic Gaussian channels: A full weak-degradability classification," New Journal of Physics, vol. 8, no. 12, p. 310, 2006, arXiv:quant-ph/0609013.

[25] V. Giovannetti, S. Guha, S. Lloyd, L. Maccone, J. H. Shapiro, and H. P. Yuen, "Classical capacity of the lossy bosonic channel: The exact solution," Physical Review Letters, vol. 92, no. 2, p. 027902, January 2004, arXiv:quant-ph/0308012.

[26] V. Giovannetti, S. Lloyd, L. Maccone, J. H. Shapiro, and B. J. Yen, "Minimum Rényi and Wehrl entropies at the output of bosonic channels," Physical Review A, vol. 70, p. 022328, August 2004, arXiv:quant-ph/0404037. [Online]. Available: http://link.aps.org/doi/10.1103/PhysRevA.70.022328

[27] R. Renner, "Security of quantum key distribution," Ph.D. dissertation, ETH Zürich, December 2005, arXiv:quant-ph/0512258.

[28] M. Tomamichel, "A framework for non-asymptotic quantum information theory," Ph.D. dissertation, ETH Zürich, March 2012, arXiv: 1203.2142 .

[29] B. R. Bardhan and M. M. Wilde, "Strong converse rates for classical communication over thermal and additive noise bosonic channels," Physical Review A, vol. 89, p. 022302, February 2014, arXiv:1312.3287. [Online]. Available: http://link.aps.org/doi/10.1103/PhysRevA.89.022302

[30] A. Nayak, "Optimal lower bounds for quantum automata and random access codes," in Proceedings of the 40th Annual Symposium on Foundations of Computer Science, New York City, NY, USA, October 1999, pp. 369-376, arXiv:quant-ph/9904093.

[31] T. M. Cover and J. A. Thomas, Elements of Information Theory. Wiley-Interscience, 2006.

[32] T. Ogawa and H. Nagaoka, "Making good codes for classical-quantum channel coding via quantum hypothesis testing," IEEE Transactions on Information Theory, vol. 53, no. 6, pp. 2261-2266, June 2007.

[33] T. Tao, Topics in Random Matrix Theory, ser. Graduate Studies in Mathematics. American Mathematical Society, 2012, vol. 132, see also http://terrytao.wordpress.com/2010/01/03/254a-notes-1-concentration-of-measure.

[34] R. Koenig, S. Wehner, and J. Wullschleger, "Unconditional security from noisy quantum storage," IEEE Transactions on Information Theory, vol. 58, pp. 1962-1984, 2012, arXiv:0906.1030.

[35] B. J. Yen and J. H. Shapiro, "Multiple-access bosonic communications," Physical Review A, vol. 72, p. 062312, December 2005, arXiv:quant-ph/0506171. [Online]. Available: http://link.aps.org/doi/10.1103/PhysRevA.72.062312 
Bhaskar Roy Bardhan was born in Kolkata, West Bengal, India. He received the B. Sc. degree in physics (honors) from St. Xavier's College, University of Calcutta, India, in 2006, the M. Sc. degree in physics from Indian Institute of Technology Guwahati, India, in 2008, and the Ph.D. degree in physics from Louisiana State University, Baton Rouge,

Currently, he is a Postdoctoral Associate at the Research Laboratory of Electronics, Massachusetts Institute of Technology, Cambridge, Massachusetts. His research interests include optical and quantum communication, quantum information theory, and quantum

Dr. Roy Bardhan is a member of the American Physical Society, the Optical Society of America, and IEEE, and has been a reviewer for the journals Physical Review Letters, Physical Review A, and Optics Letters.

Raul Garcia-Patron was born in Madrid, Spain. He received the Ph.D. degree in engineering from the Université Libre de Bruxelles, Brussels, Belgium, in 2007. He has previously hold postdoctoral positions at Massachusetts Institute of Technology and Max-Planck Institute for Quantum Optics. He currently benefits from a BELSPO return fellowship, funded by the Belgian Federal Government, in the Centre for Quantum Information and Communication at Université Libre de Bruxelles. His current research interests are in quantum Shannon theory and quantum information processing with photonic systems.

Mark M. Wilde (M'99-SM'13) was born in Metairie, Louisiana, USA. He received the Ph.D. degree in electrical engineering from the University of Southern California, Los Angeles, California, in 2008. He is an Assistant Professor in the Department of Physics and Astronomy and the Center for Computation and Technology at Louisiana State University. His current research interests are in quantum Shannon theory, quantum optical communication, quantum computational complexity theory, and quantum error correction.

Andreas Winter received a Diploma degree in Mathematics from the Freie Universität Berlin, Berlin, Germany, in 1997, and a Ph.D. degree from the Fakultät für Mathematik, Universität Bielefeld, Bielefeld, Germany, in 1999. He was Research Associate at the University of Bielefeld until 2001, and then with the Department of Computer Science at the University of Bristol, Bristol, UK. In 2003, still with the University of Bristol, he was appointed Lecturer in Mathematics, and in 2006 Professor of Physics of Information. Since 2012 he has been an ICREA Research Professor with the Universitat Autònoma de Barcelona, Barcelona, Spain. 